\title{
Clinical Decision Support for a Multicenter Trial of Pediatric Head Trauma
}

\section{Development, Implementation, and Lessons Learned}

Eric Tham ${ }^{1,2}$; Marguerite Swietlik'; Sara Deakyne'; Jeffrey M. Hoffman ${ }^{3,4}$; Robert W. Grundmeier ${ }^{5,6}$; Marilyn D. Paterno7,8; Beatriz H. Rocha ${ }^{7,8}$; Molly H Schaeffer ${ }^{9}$; Deepika Pabbathi ${ }^{9}$; Evaline Alessandrini ${ }^{10}$; Dustin Ballard ${ }^{11}$; Howard S. Goldberg ${ }^{7,8}$; Nathan Kuppermann ${ }^{12}$; Peter S. Dayan ${ }^{13}$; for the Pediatric Emergency Care Applied Research Network (PECARN)

${ }^{1}$ Children's Hospital Colorado, Aurora, CO;

2University of Colorado, Denver, CO;

${ }^{3}$ Department Pediatrics, Section of Emergency Medicine, Nationwide Children's Hospital, Columbus, $\mathrm{OH}$;

${ }^{4}$ Ohio State University College of Medicine, Columbus, $\mathrm{OH}_{\text {; }}$

${ }^{5}$ Center for Biomedical Informatics, The Children's Hospital of Philadelphia, Philadelphia, PA;

${ }^{6}$ Department of Pediatrics, Perelman School of Medicine at the University of Pennsylvania, Philadelphia PA;

${ }^{7}$ Division of General Internal Medicine, Brigham and Women's Hospital, Boston, MA;

${ }^{8}$ Harvard Medical School, Boston, MA;

IInformation Systems, Partners HealthCare System, Boston, MA;

${ }^{10}$ Cincinnati Children's Hospital Medical Center, Cincinnati, Ohio;

${ }^{11}$ Kaiser Permanente, San Rafael Medical Center; Kaiser Permanente, Division of Research, Oakland, CA;

${ }^{12}$ Departments of Emergency Medicine and Pediatrics, University of California Davis School of Medicine, Sacramento, CA;

${ }^{13}$ Pediatrics, Division of Pediatric Emergency Medicine, Columbia University College of Physicians and Surgeons, New York, NY

\section{Keywords}

Clinical decision support, clinical trial, blunt head trauma, traumatic brain injury, implementation, children

\section{Summary}

Introduction: For children who present to emergency departments (EDs) due to blunt head trauma, ED clinicians must decide who requires computed tomography (CT) scanning to evaluate for traumatic brain injury (TBI). The Pediatric Emergency Care Applied Research Network (PECARN) derived and validated two age-based prediction rules to identify children at very low risk of clinicallyimportant traumatic brain injuries (ciTBIs) who do not typically require CT scans. In this case report, we describe the strategy used to implement the PECARN TBI prediction rules via electronic health record (EHR) clinical decision support (CDS) as the intervention in a multicenter clinical trial. Methods: Thirteen EDs participated in this trial. The 10 sites receiving the CDS intervention used the Epic ${ }^{\circledR}$ EHR. All sites implementing EHR-based CDS built the rules by using the vendor's CDS engine. Based on a sociotechnical analysis, we designed the CDS so that recommendations could be displayed immediately after any provider entered prediction rule data. One central site developed and tested the intervention package to be exported to other sites. The intervention package included a clinical trial alert, an electronic data collection form, the CDS rules and the format for recommendations.

Results: The original PECARN head trauma prediction rules were derived from physician documentation while this pragmatic trial led each site to customize their workflows and allow multiple different providers to complete the head trauma assessments. These differences in workflows led to varying completion rates across sites as well as differences in the types of providers completing the electronic data form. Site variation in internal change management processes made it challenging to maintain the same rigor across all sites. This led to downstream effects when data reports were developed.

Conclusions: The process of a centralized build and export of a CDS system in one commercial EHR system successfully supported a multicenter clinical trial. 


\section{Correspondence to:}

Eric Tham, MD, MS

Seattle Children's Research Institute M/S CW8-4

2001 Eighth Avenue, Suite 400,

Seattle, WA 98121

email: eric.tham@seattlechildrens.org
Appl Clin Inform 2016; 7: 534-542

http://dx.doi.org/10.4338/ACl-2015-10-CR-0144

received: November 6, 2015

accepted: April 4, 2016

published: June 15, 2016

Citation: Tham E, Swietlik M, Deakyne S, Hoffman JM, Grundmeier RW, Paterno MD, Rocha BH, Schaeffer MH, Pabbathi D, Alessandrini E, Ballard D, Goldberg HS, Kuppermann N, Dayan PS, for the Pediatric Emergency Care Applied Research Network (PECARN). Clinical decision support for a multicenter trial of pediatric head trauma: Development, implementation, and lessons learned. Appl Clin Inform 2016; 7: 534-542 http://dx.doi.org/10.4338/ACI-2015-10-CR-0144 


\section{Introduction}

Children commonly present to emergency departments (EDs) after blunt head trauma [1]. The ED clinicians who decide which patients require emergent cranial computed tomography (CT) must weigh the potential long-term sequelae of exposure to ionizing radiation against the risks of missing clinically important traumatic brain injuries (ciTBIs) [2-8]. In order to inform this decision, investigators in the Pediatric Emergency Care Applied Research Network (PECARN) derived and validated prediction rules to identify children at very low risk of ciTBIs who do not typically require CTs [9].

By embedding the PECARN TBI prediction rules within an electronic health record (EHR) clinical decision support (CDS) system at multiple EDs, we sought to accelerate their translation and implementation into clinical practice. In this case report, we detail the methods used and lessons learned to build the EHR-based CDS system for implementing the PECARN TBI prediction rules at one site, export it, and then implement the CDS build at nine other EDs. The CDS system was the core intervention of the PECARN TBI prediction rule implementation trial (ClinicalTrials.gov Identifier NCT01453621).

\section{Methods}

The PECARN TBI prediction rule implementation trial included 13 EDs, either in the PECARN ( $n=5$ sites) or in Kaiser Permanente's Clinical Research in Emergency Services and Treatments (CREST) network ( $\mathrm{n}=8$ sites); 10 sites received CDS as the study intervention and 3 were control sites. The trial was conducted from November 2011 through June 2014. ED providers enrolled head trauma patients by entering data into the EHR. Enrollment occurred without involvement of research assistants. Institutional board approval was obtained at each site.

All sites receiving CDS in the trial used the Epic ${ }^{\oplus}$ Inpatient EHR and the Epic ${ }^{\oplus}$ ASAP ED information system (Epic Systems, Verona, WI). In order to inform the CDS system development, we conducted sociotechnical analyses at 11 of the 13 trial sites ( 2 sites joined the study after these analyses) to identify the workflow and communication, organizational factors, and human factors required to effectively implement the CDS [10]. We implemented the EHR-based CDS intervention using the vendor's internal CDS rules engine at all sites. Two sites also implemented a connection to an external web services-based CDS rules engine. The EHR-based CDS intervention was implemented using the Epic ${ }^{\circledast}$ Best Practice Advisories (BPA) CDS system.

\subsection{Provision of CDS within the ED workflow}

The informatics team and principal investigators (PIs) considered whether to present the CDS recommendation prior to or at the time of order entry. Based on the sociotechnical analysis, we chose the former strategy to allow the CDS recommendation to be presented at or near the time of the patient history and physical examination and prior to the discussion of the care plan with patient's caregivers. The sociotechnical analysis led the investigative team to conclude that if we waited until the time of order entry, then the decision whether to order a CT would have already been made.

\subsection{Development, testing and export of the EHR-based CDS system}

We considered several methods of building the CDS system, including creating one standard specification document from which all the other sites would manually build their CDS. Ultimately, we felt that exporting and importing the build from one site would result in time savings and less duplication of effort, less variation and error, and facilitate the sharing of validated testing across sites.

A lead informatics site, Children's Hospital Colorado (CHCO), developed the EHR-CDS package and exported it to participating sites. CHCO's build team consisted of a physician informaticist, a nursing informaticist, a clinical decision support analyst, and an Epic Chronicles/Cache systems administrator. The package consisted of a clinical trial alert to identify eligible patients in the EHR, an 
electronic data collection form (template) to collect the data necessary to determine the risk of ciTBI, the CDS rules, and the format of the display of the CDS recommendations.

\subsubsection{Clinical trial alert to identify eligible patients}

We developed a clinical trial alert built on Epic's BPA system and triggered by a grouping of chief complaints, customized for each site. The grouping of chief complaints was developed to maximize the sensitivity and specificity to detect children with head trauma. The chief complaint groups were based on a retrospective analysis of head injury ICD-9 codes matched to presenting chief complaints [11].

\subsubsection{EHR data collection form}

We created the EHR data collection form to capture data in a structured manner, shown in $>$ Figure 1. Structured data met the requirements necessary to provide CDS while maintaining the study validity. Structured data allowed transmission and efficient analysis at the PECARN data coordination center, located remotely. Additionally, although clinicians preferred not to collect data in a structured fashion (per our sociotechnical analysis), current clinical decision support systems are unable to use unstructured data and natural language processing to accurately extract data from clinical notes and drive CDS [10-12]. Finally, the clinicians wanted the ability to reuse the captured data in their clinical documentation, which could most consistently be accomplished using structured data.

The EHR allowed for the capture of structured data within the clinicians' clinical documentation and in specific documentation flowsheets. The documentation flowsheet was the primary documentation tool for nurses, but not physicians. Based on the sociotechnical analysis, nurses were frequently the ones who entered data at the beginning of the workflow, prior to clinician evaluation. Therefore, we chose (nursing-based) documentation flowsheets to capture the structured data, capitalizing on the EHR's flexibility to pull the same flowsheet cells into physician documentation.

While the PECARN TBI prediction rules consisted of two age-based rules, the EHR data collection form collected the combined 10 variables for both prediction rules; only the 7 relevant age-appropriate data fields were used to drive the CDS recommendations for each age group. We collected all 10 variables for all patients in order to assess baseline CT rates (before CDS) and CT rates at control sites (through the trial) without making clinicians explicitly aware of the two age-based prediction rules.

\subsubsection{CDS rules}

We developed the CDS rules from specifications authored by the Partners HealthCare informatics team. The Partners team used an iterative, four-layer design moving from narrative to computerexecutable code to create rules specifications [12, 13]. There were 2426 different possible combinations of the variables, and thus 2426 permutations of rules (based on different combinations of patient clinical variables) were created to support the vendor-specific CDS build.

\subsubsection{CDS display and wording}

Study team members finalized the CDS wording and display in an iterative, consensus-building process at all sites. The CDS content included specific recommendations if: a) the patient met the prediction rule criteria for being at very low risk of ciTBI or b) the patient was not at very low risk but met particular clinical criteria for which observation in the ED would be an acceptable strategy (see - Figure 2, for example). The CDS display also contained links to reference materials, including tables of the age-specific rules, the prediction rule publication, and the definition of ciTBI. Based on the sociotechnical analysis, the CDS display was designed to facilitate family-clinician interaction for shared decision-making by making the CDS available early in the workflow and using simple language and recommendations. Finally, the CDS displayed the most recent provider responses for each of the data elements documented in the flowsheet. This allowed the clinician who was deciding on the need for CT to reflect on the data entered, with a link to the data collection template if an update or correction to the responses was needed. 


\subsubsection{CDS testing}

The initial build required manual testing of all permutations of the CDS rules by the central site that configured the rules. A pilot site first tested a subset of the permutations. Once they validated the build and moved their build to a production environment, the implementation package was released to the other sites. Each site decided the extent of testing they would undertake. While the extent and method of testing varied at each site, there were no reported cases of incorrect CDS recommendations presented. We did not conduct formal assessments of whether incorrect CDS recommendations were presented during the trial but received no feedback of such occurrences from providers.

\subsubsection{CDS export}

The clinical trial alert, EHR data collection form, CDS rules and CDS display were packaged and exported to each site. For the baseline data collection period (prior to implementation of the CDS), the clinical trial alert with trigger criteria customized for each site and the electronic data collection form were exported. Each site chose to import the build from the lead informatics site, and required additional configuration following the import. One site chose to manually build the clinical trial alert and electronic data collection form using the imported package as a guide, based on expertise available at this site.

During the intervention phase of the trial (during which CDS was provided), the lead informatics site created another export package that contained the CDS rules and displays. This build consisted of the 13 TBI prediction risk categories and more than 250 CDS rules.

Two major changes were made to the CDS rules during the trial, requiring an update package. During the baseline data collection period, we made changes to the data collection form to capture more granular information and maintain consistency between sites of the formatting of the data fields. This change was performed by a manual process in which the lead informatics site built and tested the changes, then created a build document instructing how to make the changes manually. A second change was made after the CDS rules were implemented, as the rules specifications did not account for certain patients who had trivial trauma (as defined in the original development of the prediction rules). We manually updated the CDS rules centrally and documented the changes. This build document was then released to each site to manually update their build.

\subsection{Data Reports}

The central build site created reports in SAP Crystal Reports that extracted data from the nightly download of transactional EHR data stored in the Epic Clarity database. The reports included those study data for upload to the PECARN data center and data necessary to locally assess exclusion criteria and assess for potentially eligible patients for whom data were not completed in the EHR. These reports were customized for each site and exported. The sharing of the report with other sites was facilitated by using the same naming convention and numbering scheme for the components of the build for all sites. Each site necessarily customized the report for some of the fields, which needed to be captured from locally-existing fields.

\section{Results}

ED providers entered data for 26,856 children with head trauma; 17,703 were classified as minor head trauma, which was the primary analytic group. Of the 17,703 children with minor head trauma, 76.8\% ( $n=13,588)$ had complete age-appropriate findings documented in the flowsheets and $23.2 \%$ of patients $(n=4,115)$ had incomplete documentation of age-appropriate findings.

\subsection{Lessons learned}

The original PECARN TBI prediction rules were derived from faculty and fellow physician evaluations and documentation. However, we used a pragmatic strategy for the current trial that allowed 
nurses and resident physicians to enter data. At some sites, most data were first entered by the nurses. We emphasized to attendings that verification of data entered by nurses and residents was crucial in order to insure accuracy. We learned that, unless the attending changed at least one data point, we were unable to determine if they had actually reviewed the data prior to clinical decisionmaking.

One site decided to prevent nurses from entering data for two specific physical examination data fields pertaining to skull fractures, feeling it was not in the nurse's scope of practice (although other sites felt otherwise). This site's completion of the two skull fracture data points was $55.0 \%$ for each data point, compared to $89.0 \%$ (range $79.6-99.8 \%$ at individual sites) and $89.2 \%$ (range 80.1-99.4\%) at all other sites combined. Therefore, we learned that if more providers, such as nurses and resident physicians, are allowed to enter all data, the data will potentially be more complete.

With each site following their own internal change management processes, maintaining the same rigor across sites when changes were necessary was challenging. The lead informatics site managed a central core of knowledge as well as the EHR changes at each site. Although the build consisted of uniform naming and numbering conventions, if a site had existing use of a data element in their EHR, the site's naming and numbering conventions had to be used. This impacted the build of the data collection form as well as each portion of the CDS rules build. For instance, some sites only had one set of Glasgow Coma Scale (GCS) scores for infants, children, and adults. Other sites had two different sets of GCS scores, one for infants and children and one for adults. Thus, the CDS rules had to be changed to account for both GCS scores. Ongoing monitoring for consistency of naming and numbering of build components was crucial throughout the study. We learned that for future multicenter EHR-based studies, more rigorous change management documentation using collaborative tools such as GitHub, Microsoft Sharepoint, or JIRA Software at the study-level will facilitate maintaining changes consistently across sites.

\section{Conclusions}

We described the successful central development and implementation of a process to export and import a clinical trial alert, electronic data collection tool, and a complex CDS system to support a multicenter clinical trial using a commercial EHR. With these methods, patients could be automatically screened and enrolled into the trial without use of research assistants. These methods can be adopted for future trials, understanding the requirements and necessary resources to ensure the integrity of the CDS across sites. Future studies and implementations of CDS to support knowledge translation of decision rules will need to test the effects and capabilities among EHRs from different vendors.

\section{Conflicts of Interest}

The authors declare that they have no conflicts of interest in the research.

\section{Protection of Human Subjects}

The clinical trial was reviewed by each site's institutional review board and either was granted a waiver of consent and HIPAA authorization or was deemed non-human subjects research.

\section{Acknowledgements}

Participating centers and site investigators are listed below in alphabetical order: Cincinnati Children's Hospital and Medical Center (E. Alessandrini), Children's Hospital Boston (L. Nigrovic), Children's Hospital Colorado (L. Bajaj, M. Swietlik, E. Tham, S. Deakyne), Columbia University School of Nursing (B. Sheehan, S. Bakken), Columbus Children's Hospital (J. Hoffman), Kaiser Permanente Oakland Medical Center (D. Mark), Kaiser Permanente Roseville Medical Center (D. Vinson), Kaiser Permanente, San Rafael Medical Center (D. Ballard), Kaiser Permanente South Sacramento Medical Center (S. Offerman), Morgan Stanley Children's Hospital of New York (P. Dayan), Partners HealthCare System (H. Goldberg), University of California Davis Medical Center (N. Kuppermann, L. Tzimenatos)

We acknowledge the efforts of the following individuals participating in PECARN at the time this study was initiated. 
PECARN Steering Committee:

Voting members: Elizabeth Alpern, David Jaffe, Nathan Kuppermann, Rich Ruddy, Marc Gorelick, James Chamberlain, Rich Lichenstein, Kathleen Brown, David Monroe, Lise Nigrovic, Mike Dean, Rachel Stanley, Prashant Mahajan, Dominic Borgialli, Elizabeth Powell, John Hoyle, Lynn Babcock, Peter Dayan, Kathleen Lillis, Michael Tunik, Ellen Crain

Alternate members: Walt Schalick, Kathy Shaw, Evaline Alessandrini, Doug Nelson, Jennifer

Anders, Sally Jo Zuspan, Alex Rogers, Bema Bonsu, Maria Kwok

PECARN subcommittees:

Protocol Review and Development: David Jaffe (Chair), Kathy Shaw, Lise Nigrovic, James Chamberlain, Rachel Stanley, Elizabeth Powell, Mike Tunik, Mike Dean, Rich Holubkov

Safety and Regulatory: Walt Schalick (Co-chair), John Hoyle (Co-chair), Shireen Atabaki, Alex Rogers, David Schnadower, Maria Kwok, Heather Hibler, Kym Call

Quality Assurance: Kathy Lillis (Chair), Rich Ruddy, Evaline Alessandrini, Richard Lichenstein, Bobbe Thomas, Rachel McDuffie, Prashant Mahajan, Steve Blumberg, Jennie Wade, Rene Enriquez Feasibility and Budget: Kathy Brown (Co-chair), Sherry Goldfarb (Co-chair), Emily Kim, Doug Nelson, David Monroe, Steve Krug, Mikhail Berlyant, Ellen Crain, Sally Jo Zuspan

Grants and Publication: Marc Gorelick (Chair), Elizabeth Alpern, Jennifer Anders, Kate Shreve, Frank Moler, Dominic Borgialli, George Foltin, Lynn Babcock, Amy Donaldson

\section{Funding}

American Recovery and Reinvestment Act-Office of the Secretary (ARRA OS): Grant \#S02MC19289-01-00. PECARN is supported by the Health Resources and Services Administration (HRSA), Maternal and Child Health Bureau (MCHB), Emergency Medical Services for Children (EMSC) Program through the following cooperative agreements: U03MC00001, U03MC00003, U03MC00006, U03MC00007, U03MC00008, U03MC22684, and U03MC22685. 


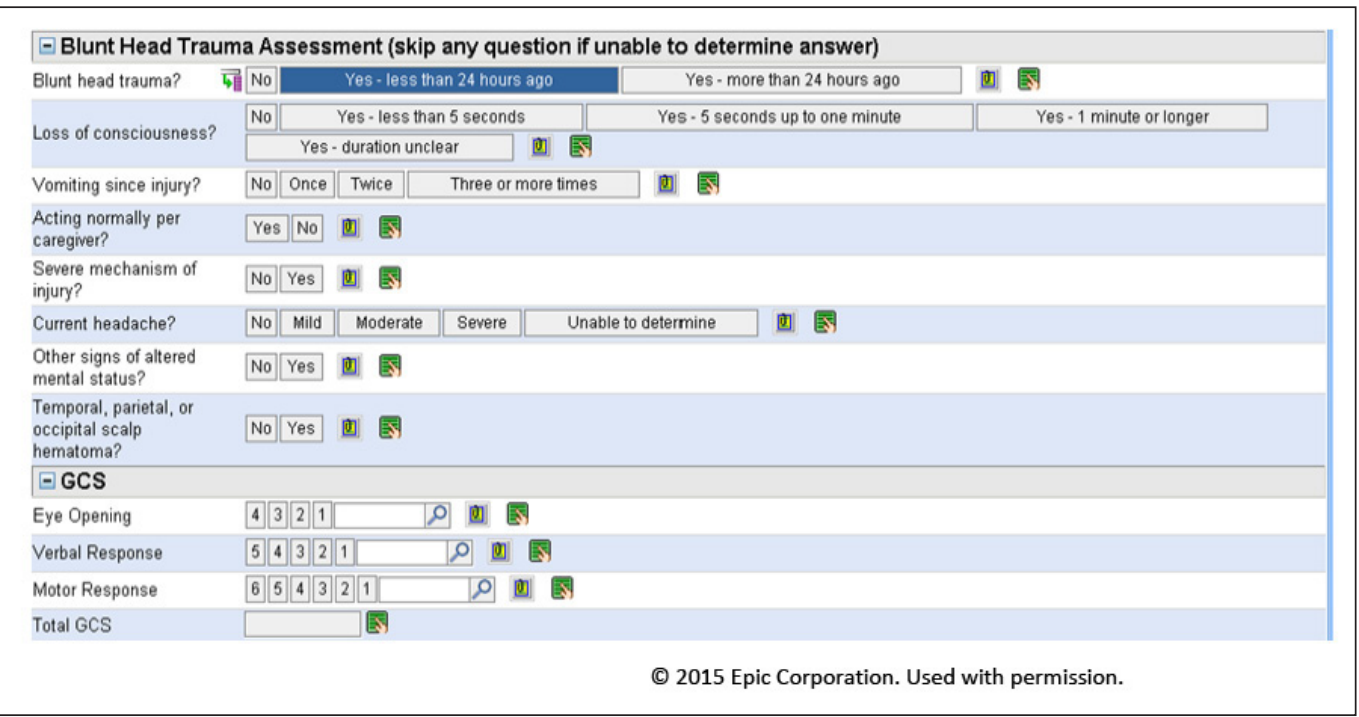

Fig. 1 EHR-based data collection form used for clinical trial

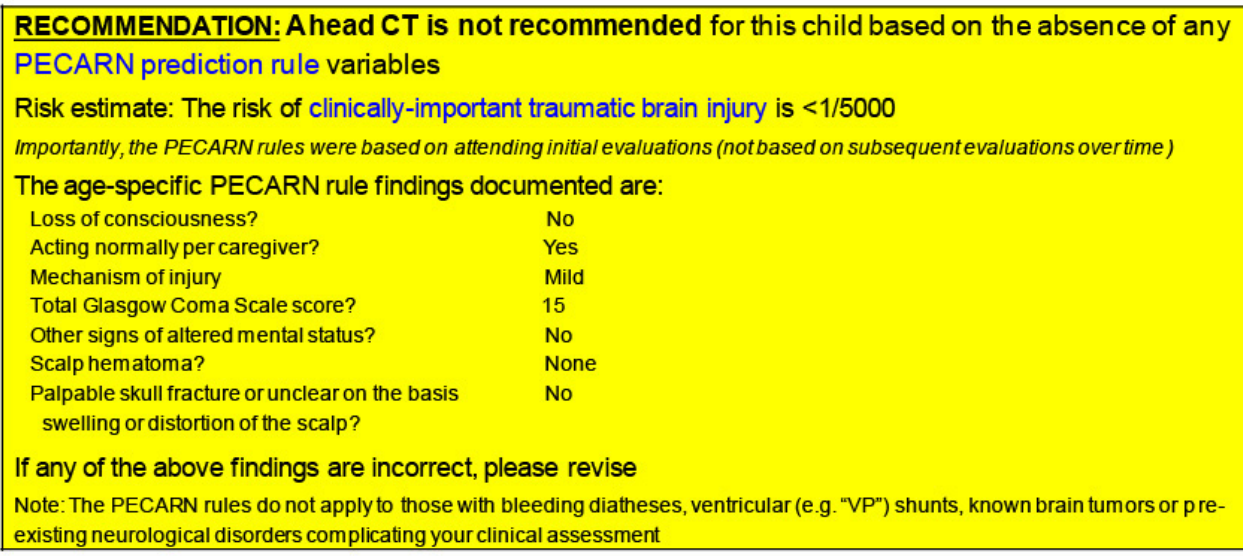

* All decision support provided followed a similar format: recommendation, risk estimate, documented findings in head trauma template, ability to revise, and specific information regarding application of prediction rules.

Fig. 2 Example of display of clinical decision support* 


\section{References}

1. Centers for Disease Control and Prevention. Rates of TBI-related Emergency Department Visits by Age Group- United States, 2001-2010 2014 [updated February 24, 2014; cited 2014 October 22]. Available from: http://www.cdc.gov/traumaticbraininjury/data/rates_ed_byage.html.

2. Brenner D. Estimating cancer risks from pediatric CT: going from the qualitative to the quantitative. Pediatric Radiology 2002; 32(4): 228-231. doi: 10.1007/s00247-002-0671-1.

3. Brenner DJ, Elliston CD, Hall EJ, Berdon WE. Estimated risks of radiation-induced fatal cancer from pediatric CT. American Journal of Roentgenology 2001; 176(2): 289-296. doi: 10.2214/ajr.176.2.1760289.

4. Pearce MS, Salotti JA, Little MP, McHugh K, Lee C, Kim KP, Howe NL, Ronckers CM, Rajaraman P, Craft AW, Parker L, Berrington de González A. Radiation exposure from CT scans in childhood and subsequent risk of leukaemia and brain tumours: a retrospective cohort study. The Lancet 2012; 380(9840): 499-505. doi: http://dx.doi.org/10.1016/S0140-6736(12)60815-0.

5. Hennelly KE, Mannix R, Nigrovic LE, Lee LK, Thompson KM, Monuteaux MC, Proctor M, Schutzman S. Pediatric traumatic brain injury and radiation risks: A clinical decision analysis. The Journal of Pediatrics 2013; 162(2): 392-397. doi: http://dx.doi.org/10.1016/j.jpeds.2012.07.018.

6. Miglioretti DL, Johnson E, Williams A, Greenlee RT, Weinmann S, Solberg LI, Feigelson HS, Roblin D, Flynn MJ, Vanneman N, Smith-Bindman R. The use of computed tomography in pediatrics and the associated radiation exposure and estimated cancer risk. JAMA Pediatrics 2013; 167(8): 700-707. doi: 10.1001/jamapediatrics.2013.311.

7. Mathews JD, Forsythe AV, Brady Z, Butler MW, Goergen SK, Byrnes GB, Giles GG, Wallace AB, Anderson PR, Guiver TA, McGale P, Cain TM, Dowty JG, Bickerstaffe AC, Darby SC. Cancer risk in 680,000 people exposed to computed tomography scans in childhood or adolescence: data linkage study of 11 million Australians. BMJ 2013; 346: f2360. doi: 10.1136/bmj.f2360.

8. Nishijima DK, Yang Z, Urbich M, Holmes JF, Zwienenberg-Lee M, Melnikow J, Kuppermann N. Cost-effectiveness of the PECARN Rules in Children With Minor Head Trauma. Annals of Emergency Medicine 2015; 65(1): 72-80 e6.

9. Kuppermann N, Holmes JF, Dayan PS, Hoyle JD, Atabaki SM, Holubkov R, Nadel FM, Monroe D, Stanley RM, Borgialli DA, Badawy MK, Schunk JE, Quayle KS, Mahajan P, Lichenstein R, Lillis KA, Tunik MG, Jacobs ES, Callahan JM, Gorelick MH, Glass TF, Lee LK, Bachman MC, Cooper A, Powell EC, Gerardi MJ, Melville KA, Muizelaar JP, Wisner DH, Zuspan SJ, Dean JM, Wootton-Gorges SL. Identification of children at very low risk of clinically-important brain injuries after head trauma: a prospective cohort study. The Lancet 2009; 374(9696): 1160-1170. doi: http://dx.doi.org/10.1016/S0140-6736(09)61558-0.

10. Sheehan B, Nigrovic LE, Dayan PS, Kuppermann N, Ballard DW, Alessandrini E, Bajaj L, Goldberg H, Hoffman J, Offerman SR, Mark DG, Swietlik M, Tham E, Tzimenatos L, Vinson DR, Jones GS, Bakken S. Informing the design of clinical decision support services for evaluation of children with minor blunt head trauma in the emergency department: A sociotechnical analysis. Journal of Biomedical Informatics 2013; 46(5): 905-913. doi: 10.1016/j.jbi.2013.07.005.

11.Deakyne SJ, Bajaj L, Hoffman J, Alessandrini E, Ballard DW, Norris R, Tzimenatos L, Swietlik M, Tham E, Grundmeier RW, Kuppermann N, Dayan for the Pediatric Emergency Care Applied Research N. Development, evaluation and implementation of chief complaint groupings to activate data collection: A multicenter study of clinical decision support for children with head trauma. Applied Clinical Informatics 2015; 6(3): 521-535. doi: 10.4338/ACI-2015-02-RA-0019.

12. Boxwala AA, Rocha BH, Maviglia S, Kashyap V, Meltzer S, Kim J, Tsurikova R, Wright A, Paterno MD, Fairbanks A, Middleton B. A multi-layered framework for disseminating knowledge for computer-based decision support. Journal of the American Medical Informatics Association 2011; 18(Suppl. 1): i132-i139. doi: 10.1136/amiajnl-2011-000334.

13. Goldberg HS, Paterno MD, Rocha BH, Schaeffer M, Wright A, Erickson JL, Middleton B. A highly scalable, interoperable clinical decision support service. Journal of the American Medical Informatics Association 2014; 21(e1): e55-e62. doi: 10.1136/amiajnl-2013-001990. 